\title{
Padé Approximation Based on Orthogonal Polynomial
}

\author{
Bo Wu and Youhua Qian * \\ College of Mathematics, Physics and Information Engineering, Zhejiang Normal University, Jinhua, China \\ *Corresponding author
}

\begin{abstract}
For the sake of solving the problem of nonlinear approximation better, we mainly use the Padé approximation under the orthogonal basis function in this paper. Through figuring out the orthogonal basis function for a given product, we can obtain the $[m, n]$ order Padé approximation of arbitrary rational polynomial. At the same time, specific examples are given to verify the effectiveness of the method.
\end{abstract}

Keywords-Padé approximation; orthogonal basis function; inner product

\section{INTRODUCTION}

As is known to all, the purpose of approximation is to use simple functions to approximate more complex functions, polynomial is a common approximation tool. With the rapid development of electronic technology, the polynomial approximation has been rapidly developed and widely used. Although the polynomial is a very good approximation function, the polynomial approximation has its deficiency ${ }^{[1]}$. For some singular functions, the polynomial approximation can not reach a good approximation effect. However, Padé approximation as a generalization of polynomial, it can achieve a favorable effect near poles. Padé approximation is a special type of rational function approximation ${ }^{[2]}$. Gradually being recognized by people, it is widely used in practical problem, especially in many physical problems. Since the last century 70s, the theory and application of Padé approximation has been developed rapidly, more and more scientists have paid attention to it and carried out a lot of research. In 1992, Bustamante consider the Nikishin system of analytic function based on Hermit-Padé approximations and prove the convergence of the method, furthermore, multipoint Padé approximations has played important role in proof ${ }^{[3]}$. In 2007, Gonchar et al. discuss the system of Markov function with Hermit-Padé approximations, then obtain the certain equilibrium problems of potential theory with respect to vector potential $^{[4]}$. In 2015, López Lagomasino et al. deal with the convergence properties of the Hermit-Padé approximations, moreover, they furnish Stieltjes type and Markov theorems on the convergence of Hermit-Padé approximations for Nikishin system of function $^{[5]}$. In 2006, Assche et al. provide a short presentation to Hermit-Padé approximations and Padé approximation. In addition, they demonstrate some convergence properties of the approximations ${ }^{[6]}$. In 2009, Liu et al. research the nonlinear differential-difference equations by the adomian decomposition method and Padé approximation, the numerical indicated that we can achieve the approximation solution with faster convergence rate and higher precision than use adomian decomposition method ${ }^{[7]}$. In 2010, Betancourt et al. explore the power system oscillations in the way of adomian method and Padé approximation. They solve the differential equation by the adomian method and receive oscillations characteristics with Padé approximation ${ }^{[8]}$. In 2010, Lisa Lorentzen explore the Padé approximation and continued fractions, in addition, they provide the convergence of continued fractions and obtain fast and steady convergence of approximation ${ }^{[9]}$. In 2012, Natori research a design method of time-delay by taking advantage of Padé approximation, by this means, detailed pole position can be obtained ${ }^{[10]}$. In 2013, by solving linear and nonlinear fractional partial differential equation, Turut et al, put forward multivariate Padé approximation. Compared with variational iteration method, this methods are very valid and expedient ${ }^{[11]}$. In 2015, Dogonchi et al. Discuss the non-stationary motion of the perpendicularly falling in incompressible Newtonian medium $^{[12]}$. The speed and acceleration are acquired via utilizing Padé approximation and differential transformation method. As a matter of fact, there is close connection between Padé approximation and some branches of mathematics. For instance, approximation theory, differential equation ${ }^{[13][14]}$ and analytic function, etc. It has achieved prominent result in quantum field theory, numerical analysis, control theory and other domain of natural sciences.

\section{PADÉ APPROXIMATION BASED ON ORTHOGONAL POLYNOMIAL}

\section{A. Approximation Theory}

The classical Padé approximation method is defined as follows:

Let

$$
f(x)=\sum_{j=0}^{\infty} a_{j} x^{j}, a_{0} \neq 0
$$

where the polynomial $P_{L}(x)$ and $Q_{M}(x)$ have no common factor. In the meantime, their power is not more than $\mathrm{L}, \mathrm{M}$ respectively. Their coefficients are determined by the following equation ${ }^{[15]}$ : 


$$
f(x)-\frac{P_{L}(x)}{Q_{M}(x)}=o\left(x^{L+M+1}\right)
$$

Assuming $Q_{M}(0)=0$,we call rational factor $P_{L}(x) / Q_{M}(x)$ as the $(L, M)$ order Padé approximation of the function $f(x)$

$$
[L / M]=P_{L}(x) / Q_{M}(x),
$$

which

$$
\begin{aligned}
& P_{L}(x)=p_{0}+p_{1} x+\cdots+p_{L} x^{L} \\
& Q_{M}(x)=1+q_{1} x+\cdots+q_{M} x^{M} .
\end{aligned}
$$

\section{B. Orthogonal Basis Functions}

Let $f(x), g(x)$ are continuous function on $[a, b]$, if

$$
\int_{a}^{b} f(x) g(x) d x=0
$$

then $f(x), g(x)$ is called orthogonal on $[a, b]$.

We call such a function set is orthogonal functions, if

$$
\int_{a}^{b} \varphi_{m}(x) \varphi_{n}(x) d x=\left\{\begin{array}{l}
0, m \neq n \\
1, m=n
\end{array} .\right.
$$

Let $\left\{\phi_{n}(x) \mid n=1,2, \cdots\right\}=H$ as a set of orthogonal function system, and the product of any two basis functions can be expressed as a linear combination of $\mathrm{H}$.

C. Padé Approximation Concept Based on Orthogonal Basis Functions

$$
\text { We call } \frac{\sum_{i=1}^{m} a_{i} \varphi_{i}(x)}{1+\sum_{j=2}^{n} b_{j} \varphi_{j}(x)} \text { is }[m, n] \text { order Padé }
$$

approximation of the $f(x)$, if

$$
\left\langle f(x)\left(1+\sum_{j=2}^{n} b_{j} \varphi_{j}(x)\right)-\sum_{i=2}^{m} a_{i} \varphi_{i}(x), \varphi_{k}(x)\right\rangle=0
$$

where $k=1,2, \cdots m+n-1$.

In the polynomial orthogonal function system, there are the following conclusions: $\left\{\varphi_{k}(x) k=1,2, \cdots\right\}$ is the polynomial orthogonal functions, for any $\varphi_{i}(x), \varphi_{j}(x) \in\left\{\varphi_{k}(x) \mid k=1,2, \cdots\right\}$ we can know that

$$
\varphi_{i}(x) \varphi_{j}(x)=\sum_{k=1}^{i+j} d_{k} \varphi_{k}(x)
$$

For the function $f(x)$ letting $f(x)=\sum_{i=1}^{\infty} c_{k} \varphi_{k}(x)$, we can obtain polynomial Padé approximation of the function $f(x)$ :

$$
\frac{\sum_{i=1}^{m} a_{i} f_{i}(x)}{1+\sum_{j=2}^{n} b_{j} f_{j}(x)} \approx f(x)
$$

thus, we can obtain that

$$
\left\langle f(x)\left(1+\sum_{j=2}^{n} b_{j} \varphi_{j}(x)\right)-\sum_{i=1}^{m} a_{i} \varphi_{i}(x), \varphi_{t}(x)\right\rangle=0
$$

$$
\left\langle\sum_{p=1}^{N} u_{p} \varphi_{p}(x), \varphi_{t}(x)\right\rangle=0
$$

Therefore, according to the above process, we can obtain the Padé approximation of each order of rational polynomial function.

\section{Padé Approximation Based on Polynomial Orthogonal} Basis Functions

We defined the following two different inner product as follows:

$$
\begin{aligned}
& \left(u_{i}(x), u_{j}(x)\right)=\int_{0}^{1} u_{i}(x) u_{j}(x)+u_{i}^{\prime}(x) u_{j}^{\prime}(x) d x \\
& \left(u_{i}(x), u_{j}(x)\right)= \\
& \int_{0}^{1} u_{i}(x) u_{j}(x)+2 u_{i}^{\prime}(x) u_{j}^{\prime}(x)+u_{i}^{\prime \prime}(x) u_{j}^{\prime \prime}(x) d x
\end{aligned}
$$

According to the definition of the inner product, we can receive the orthogonal basis function. 


$$
\left(u_{i}(x), u_{j}(x)\right)=\int_{0}^{1} u_{i}(x) u_{j}(x)+u_{i}^{\prime}(x) u_{j}^{\prime}(x) d x
$$

Letting

$$
f_{1}(x)=1, \quad f_{2}(x)=x+b .
$$

According to the above definition of the inner product, we can obtain that:

$$
\int_{0}^{1}(x+a) d x=0
$$

then,

$$
b=-\frac{1}{2} \text { and } f_{2}(x)=x-\frac{1}{2}
$$

According to the above method, we can get:

$$
\begin{aligned}
f_{3}(x)= & x^{2}-x+\frac{1}{6} \\
f_{4}(x)= & x^{3}-\frac{3}{2} x^{2}+\frac{33}{65} x-\frac{1}{260} \\
f_{5}(x)= & x^{4}-2 x^{3}+\frac{513}{427} x^{2}-\frac{86}{427} x+\frac{1}{4270} \\
f_{6}(x)= & x^{5}-\frac{5}{2} x^{4}+\frac{35900}{16749} x^{3}-\frac{7985}{11166} x^{2} \\
& +\frac{5605}{78162} x-\frac{1}{468972}, \\
f_{7}(x)= & x^{6}-3 x^{5}+\frac{113975}{341902} x^{4}-\frac{285020}{170951} x^{3} \\
& +\frac{61115}{170951} x^{2}-\frac{8161}{341902} x+\frac{1}{14359884}, \\
f_{8}(x)= & x^{7}-\frac{7}{2} x^{6}+\frac{46022193}{9642373} x^{5}-\frac{12273875}{38569492} x^{4} \\
& +\frac{112536235}{106066103} x^{3}-\frac{33776253}{212132206} x^{2} \\
& +\frac{219527}{28927119} x-\frac{1}{254558642},
\end{aligned}
$$

By the same method, we can also get another orthogonal basis function under the definition of the inner product.

E. Examples

The $[m, n]$ order Padé approximation of the function $e^{-x}$ :

Under inner product

$$
\left(u_{i}(x), u_{j}(x)\right)=\int_{0}^{1} u_{i}(x) u_{j}(x)+u_{i}^{\prime}(x) u_{j}^{\prime}(x) d x
$$

Choosing $\left\{f_{i}(x) \mid i=1,2, \cdots 8\right\}$ as the base, we can get polynomial Padé approximation of the function $e^{-x}$ :

$$
\frac{\sum_{i=1}^{m} a_{i} f_{i}(x)}{1+\sum_{j=2}^{n} b_{j} f_{j}(x)} \approx e^{-x}
$$

[2,1] order Padé approximation:

$$
\frac{a_{1} f_{1}(x)+a_{2} f_{2}(x)}{1} \approx e^{-x} .
$$

From (6) we can obtain

$$
\left\langle e^{-x}-\sum_{i=1}^{2} a_{i} f_{i}(x), f_{k}(x)\right\rangle=0
$$

Then,

$$
\begin{aligned}
& a_{1}=1-\frac{1}{e}, a_{2}=-\frac{6}{13 e}-\frac{6}{13}, \\
& R_{2,1}(x)=\frac{a_{1} f_{1}(x)+a_{2} f_{2}(x)}{1} .
\end{aligned}
$$

[2,2] order Padé approximation:

$$
\frac{a_{1} f_{1}(x)+a_{2} f_{2}(x)}{1+b_{2} f_{2}(x)} \approx e^{-x},
$$

therefore, 


$$
\left\langle e^{-x}\left(1+b_{2} f_{2}(x)\right)-\sum_{i=1}^{2} a_{i} f_{i}(x), f_{k}(x)\right\rangle=0
$$

then,

$$
\begin{aligned}
& a_{1}=\frac{24}{25}-\frac{24}{25 e}, \\
& a_{2}=-\frac{408 / e+384 / e^{2}-192}{975 / e-325}, \\
& b_{2}=-\frac{2 / e-2}{75 / e-25}, \\
& R_{2,2}(x)=\frac{a_{1} f_{1}(x)+a_{2} f_{2}(x)}{1+b_{2} f_{2}(x)} .
\end{aligned}
$$

In the same way, we can get the coefficients of the $[m, n]$ order Padé approximation of the function $e^{-x}$.

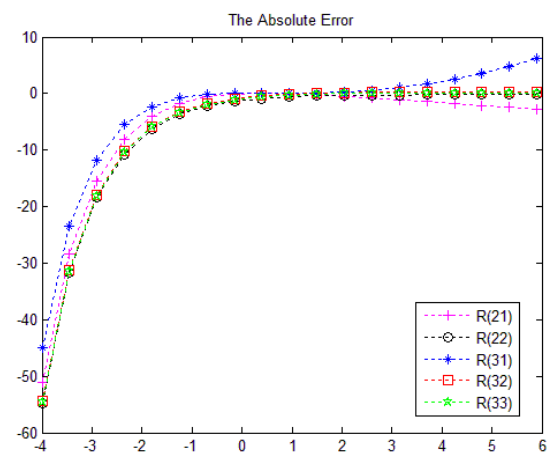

FIGURE I. THE ABSOLUTE ERROR CURVE OF [2,1], [2,2], [3,1],

\section{$[3,2],[3,3]$ ORDER PADÉ APPROXIMATION OF $e^{-x}$}

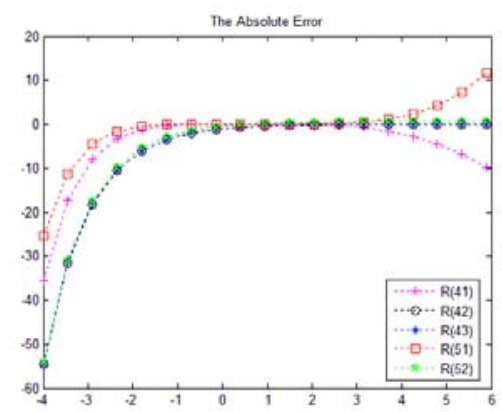

FIGURE II. THE ABSOLUTE ERROR CURVE OF $[4,1],[4,2],[4,3]$,

\section{CONCLUSION}

A number of problem can be attributed to the solution of nonlinear systems in engineering, mechanics and some domain of natural science. Padé approximation is frequently used for solving nonlinear problem, it has received extensive concern of academic circle. In this paper, we present the correlative conception of Padé approximation. Meanwhile, concrete examples are provided to examine the validity of Padé approximation.

\section{ACKNOWLEDGMENT}

The authors gratefully acknowledge the support of the National Natural Science Foundations of China (NNSFC) through grant Nos. 11202189 and 11572288 .

\section{REFERENCES}

[1] G A. Baker. Essentials of Padé approximations. Academic Press, 1975.

[2] G A. Baker and P. Graves-Morris. Padé approximations. Part 1: Basic theory. Encyclopedia of Mathematics and its applications, AddisonWesley, Reading, 1981.

[3] Z. H. Bustamante, Hermite-Padé approximations for Nikishin systems of analytic functions. Mathematics Sbornik, 1992, pp. 117-138.

[4] A. A. Gonchar, E. A. Rakhmanov, and V. N. Sorokin. Hermite-Padé approximations for systems of Markov-type functions. Russian Academy of Sciences Sbornik Mathematics , 2007, pp. 33-58.

[5] G. López Lagomasino et al. On the convergence of type 1 Hermite-Padé approximants. Advances in Mathematics, 2015, pp. 124-148.

[6] W. V. Assche, Padé and Hermite-Padé approximation and orthogonality. Surveys in Approximation Theory, 2006, pp. 61-91.

[7] Liu Yan-Ming et al. Adomian decomposition method and Padé approximation for nonlinear differential-difference equations. Communications in Theoretical Physics, 2009, pp. 581-587.

[8] R. J. Betancourt, et al. Analysis of inter-area oscillations in power systems using Adomian-Padé approximation method. IEEE/IAS International Conference on Industry Applications, 2010, pp.1-6.

[9] Lisa Lorentzen, Padé approximation and continued fractions. Applied Numerical Mathematics, 2010,pp. 1364-1370.

[10] Natori, Kenji, A design method of time-delay systems with communication disturbance observer by using Padé approximation. IEEE International Workshop on Advanced Motion Control, 2012, pp. 1 -6 .

[11] V. Turut and N. Güzel, On solving Partial Differential Equations of Fractional Order by Using the Variational Iteration Method and Multivariate Padé Approximations. European Journal of Pure \& Applied Mathematics, 2013.

[12] A. S. Dogonchi, et al. Non-spherical particles sedimentation in an incompressible Newtonian medium by Padé approximation. Powder Technology , 2015, pp. 248-256.

[13] A. F. Vakakis and M. F. A. Azeez, Analytic approximation of the homoclinic orbits of the Lorenz system at $\sigma=10, b=8 / 3$ and $\rho=13.926$ Nonlinear Dynamics, 1998, pp. 245-257.

[14] Y. V. Mikhlin, Analytic construction of homoclinic orbits of two- and three-dimensional dynamical systems. Journal of Sound and Vibration, 2000, pp. 971-983.

[15] M. Prevost, Diophantine approximations using Padé approximations. Journal of Computational and Applied Mathematics, 2000, pp. 231-50.

\section{$[5,1],[5,2]$ ORDER PADÉ APPROXIMATION OF $e^{-x}$}

